\title{
Personality goes a long a way: an interhemispheric connectivity study
}

\section{Sylco S. Hoppenbrouwers ${ }^{1 \dagger}$, Faranak Farzan ${ }^{2 \dagger}$, Mera S. Barr ${ }^{2}$, Aristotle N. Voineskos ${ }^{2}$, Dennis J.L.G. Schutter', Paul B. Fitzgerald ${ }^{3}$ and Zafiris J. Daskalakis ${ }^{2 *}$}

1 Department of Experimental Psychology, Utrecht University, Utrecht, Netherlands

2 Schizophrenia Program, Centre for Addiction and Mental Health, Toronto, ON, Canada

${ }^{3}$ Monash Alfred Psychiatry Research Centre, The Alfred and Monash University School of Psychology and Psychiatry, Melbourne, VIC, Australia

\section{Edited by:}

Ziad Nahas, Medical University of South Carolina, USA

Reviewed by:

Malek Bajbouj, Charité, Germany

Paul Croarkin, UT Southwestern

Medical Center, USA

${ }^{*}$ Correspondence:

Zafiris J. Daskalakis, Centre for

Addiction and Mental Health, 250

College Street, Toronto, ON, Canada.

e-mail:jeff_daskalakis@camh.net

${ }^{\dagger}$ Sylco S. Hoppenbrouwers and

Faranak Farzan have contributed equally to this work.

Throughout the development of psychology the delineation of personality has played a central role. Together with the NEO-PI-R, a questionnaire derived from the Five Factor Model of Personality, and recent advances in research technology it is now possible to investigate the relationship between personality features and neurophysiological brain processes. The NEO-FFI, the short version of the NEO-PI-R, reliably measures five main personality traits: Neuroticism, Extraversion, Openness to experience, Agreeableness, and Conscientiousness. As behavior and some psychiatric disorders have been related to interhemispheric connectivity, the present study used the combination of transcranial magnetic stimulation (TMS) and electroencephalography (EEG) to measure frontal interhemispheric connectivity and its association with personality as indexed by the NEO-FFI. Results demonstrated that prefrontal interhemispheric connectivity between the left and right dorsolateral prefrontal cortex correlates with Agreeableness in healthy subjects. This is the first study to relate personality features to interhemispheric connectivity through TMS-EEG and suggests that Agreeableness relates to the effectiveness of prefrontal communication between hemispheres.

Keywords: transcranial magnetic stimulation, electroencephalography, interhemispheric connectivity, NEO-PI-R, agreeableness

\section{INTRODUCTION}

Personality can be defined as the dynamic organization of psychophysical systems that create the person's characteristic patterns of thoughts, feelings, and behavior (Allport, 1961). Similar to IQ, personality is a robust predictor of life outcome as it reliably predicts divorce, mortality, and occupational attainment (McAdams and Olson, 2010). One of the major advances in personality research has been the development of the NEO-PI-R, a questionnaire derived from the Five Factor Model (FFM) of personality that has become a prevalent assessment tool for personality (McCrae, 1991; McCrae et al., 2005). The NEO-PI-R, and its short version the NEO Five Factor Inventory (NEO-FFI) consists of the five factors: Neuroticism, Extraversion, Openness to experience, Agreeableness, and Conscientiousness.

Personalities develop until late adolescence and are influenced by parental care, contact with peers, and the predominant culture. Although most measures of personality remain relatively stable in adulthood aspects of personality may change in later life (Roberts et al., 2006). In the last decade, advances in both research technology and the development of reliable indexes of personality have uncovered relationships between personality and certain brain processes. For instance, Tauscher et al. (2001) reported an inverse relationship between prefrontal serotonin receptor binding potential and anxiety, a constituent of the neuroticism scale. In addition, neuroticism has been associated with frontolimbic serotonin receptor binding, a correlation that was mainly driven by the subfactors vulnerability and anxiety (Frokjaer et al., 2008). Arguably, the association of serotonin receptor functioning with vulnerability and anxiety that can predispose to major depressive disorder (MDD) may explain the efficacy of selective serotonin reuptake inhibitors (SSRI) (Tauscher et al., 2001; Frokjaer et al., 2008). In a transcranial magnetic stimulation (TMS) study, neuroticism was found to positively correlate with cortical inhibition over the motor cortex (Wassermann et al., 2001). TMS is a relatively new research tool that allows for non-invasive investigation of brain areas and the communication between brain areas. Originally confined to the motor cortex, TMS has recently been combined with electroencephalography (EEG) enabling measurement of cortical inhibition from non-motor areas such as the dorsolateral prefrontal cortex (DLPFC) (Daskalakis et al., 2008a). Importantly, cortical inhibition measured in the DLPFC has been associated with working memory performance (Daskalakis et al., 2008b).

Through the combination of TMS and EEG, the connectivity between hemispheres can also be assayed in cortical regions more relevant to human affect, cognition and behavior such as the prefrontal cortex. Recently, we reported that TMS-induced interhemispheric signal propagation (ISP) correlates with the microstructural integrity of fiber bundles that constitute the corpus callosum showing that a higher integrity of the callosal fibers is associated with lower ISP (Voineskos et al., 2010). ISP is defined as the ratio of right to left cortical evoked activity (Voineskos et al., 2010). Specifically, ISP induced by single pulse TMS over the motor cortex significantly correlated with fractional anisotropy (FA) of callosal motor fibers but not with FA of the genu. FA represents the extent to which parts of the cytoskeleton including axons and cell bodies restrict the diffusion of water molecules 
(Beaulieu, 2002). Conversely, ISP induced by prefrontal single pulse TMS was significantly correlated with FA of the genu but not with callosal motor fibers suggesting that interhemispheric connectivity as measured with TMS-EEG is mediated by interhemispheric callosal pathways (Voineskos et al., 2010).

Functionally, interhemispheric connectivity represents a neurophysiological mechanism that allows the hemispheres to interact enabling hemispheric lateralization and specialization of functions such as language (Doron and Gazzaniga, 2008). However, affect has also been shown to relate to hemispheric lateralization (van Honk and Schutter, 2006; Schutter et al., 2008) while human aggressive behavior has been associated with asymmetric interhemispheric connectivity (Hofman and Schutter, 2009). In addition, deficits in interhemispheric connectivity have been argued to give rise to certain psychiatric disorders such as attention deficit hyperactivity disorder (ADHD) (Hoeppner et al., 2008) and MDD (Bajwa et al., 2008). Taken together, these studies suggest that interhemispheric connectivity may underlie various cognitive, affective, and behavioral functions that together constitute a personality style. In the present study, therefore, we sought to investigate the relationship between prefrontal interhemispheric connectivity as measured with TMS and EEG and the construct of personality as indexed by the NEO-FFI.

\section{MATERIALS AND METHODS PARTICIPANTS}

Ten right-handed healthy subjects (mean age $=37.8 \pm 7.8$, range $=$ 27-48 years; 4 males, 6 females) were recruited via advertisements or self-referral, and psychopathology was ruled out through the personality assessment screener (PAS; Psychological Assessment Resources, Inc). Exclusion criteria included a self-reported medical illness or a history of drug or alcohol abuse. Handedness was confirmed using the Edinburgh Handedness Inventory (Oldfield, 1971). We also administered a TMS safety screening and demographic questionnaire. Subjects had no history of seizure, brain surgery or stroke, and had never experienced external head trauma. The average weight and height across subjects were 146.9 $\pm 35.3 \mathrm{lbs}$ ( range $=102-$ $210 \mathrm{lbs}$ ) and $5^{\prime} 7^{\prime \prime} \pm 5^{\prime \prime}$ (range $=5^{\prime} 1^{\prime \prime}-6^{\prime} 4^{\prime \prime}$ ), respectively. Subjects had $16 \pm 2$ years of education, and the mean handedness score (laterality quotient) was $89 \pm 15$ (range $=+56$ to +100 ) across subjects. Finally, the NEO-FFI was administered in all subjects. The protocol was approved by the Research Ethics Board of the Centre for Addiction and Mental Health and all subjects gave their written informed consent in accordance with the declaration of Helsinki.

\section{TRANSCRANIAL MAGNETIC STIMULATION}

Single monophasic TMS pulses were administered to the left motor cortex and left DLPFC using a 7-cm figure-of-eight coil, and a Magstim 200 stimulator (Magstim Company Ltd, UK). In motor cortex, the TMS coil was placed at the optimal position for eliciting motor evoked potentials (MEPs) from the right abductor pollicis brevis (APB) muscle, which typically corresponded to a region between FC3 and C3 electrodes on the 10-20 EEG system (Herwig et al., 2003). Localization of the DLPFC was achieved through neuronavigation techniques using the MINIBIRD system (Ascension Technologies, USA) and MRIcro/reg software using a T1-weight MRI scan obtained for each subject with seven fiducial markers in place. Stimulation was directed at the junction of the middle and anterior one-third of the middle frontal gyrus (Talairach Co-ordinates $(x, y, z)=(50,30,36))$ corresponding with posterior regions of Brodmann Area (BA) 9 which overlap with the superior section of BA46, as previously described (Rusjan et al., in press). The optimal position was marked on the EEG cap to ensure identical placement of the coil, and the handle of the coil pointed backward, perpendicular to the presumed direction of the central sulcus, approximately $45^{\circ}$ to the mid-sagittal line.

The TMS intensity was determined at the beginning of each experiment. For each subject, resting motor threshold (RMT) was defined as the minimum stimulus intensity that elicits a MEP of more than $50 \mu \mathrm{V}$ in five of ten trials (Rossini et al., 1994). This corresponded to $39.1 \pm 6.1 \%$ of stimulator output. The suprathreshold intensity was set to elicit an average MEP of $1 \mathrm{mV}$ peak-to-peak amplitude upon delivery of 20 pulses over the motor cortex; this corresponded to $63.1 \pm 12.1 \%$. A total of one hundred suprathreshold stimuli, with an interstimulus interval of $5 \mathrm{~s}$, were delivered to each cortical region (i.e., motor cortex and DLPFC).

\section{ELECTROMYOGRAPHY}

To capture EMG, two disposable disk electrodes were placed over the right APB in a tendon-belly arrangement. MEPs were band-pass filtered ( $2 \mathrm{~Hz}$ to $5 \mathrm{kHz}$ ), digitized at $5 \mathrm{kHz}$, and collected through commercially available software Signal (Cambridge Electronics Design, UK).

\section{ELECTROENCEPHALOGRAPHY}

To evaluate TMS-induced cortical evoked potentials, EEG was acquired through Synamps2 EEG system (Compumedics, Charlotte, USA) using a 64-channel EEG cap. In order to monitor eye movement artifacts, four additional electrodes were placed on the outer side of each eye, and above and below the left eye. All electrodes were referenced to an electrode placed on the vertex posterior to the $\mathrm{Cz}$ electrode. EEG signals were recorded with filters at DC to $100 \mathrm{~Hz}$ at $20 \mathrm{kHz}$ sampling rate, which was shown to minimize the TMS-related artifacts (Daskalakis et al., 2008). The EEG recordings were analyzed as previously described (Daskalakis et al., 2008; Farzan et al., 2009), that is, for each subject and recording site, artifact free post-stimulus cortical evoked potentials were extracted and averaged.

\section{TMS-INDUCED ISP}

To quantify the propagation of TMS induced cortical evoked activity, the average cortical evoked potentials were band pass filtered $(1-50 \mathrm{~Hz})$ and TMS-induced ISP was calculated through Eq. 1 (Voineskos et al., 2010). To evaluate TMS-induced ISP in the motor cortex, C3 (left hemisphere) and C4 (right hemisphere) electrodes were used, which are the electrodes closest to the optimal site of APB activation through TMS. In the DLPFC, the recording electrodes of interest were AF3 (left hemisphere) and AF4 (right hemisphere), which optimally represent the overlap of BA9 and 46. In the left hemisphere, the area under the rectified curve was obtained between 50 and $150 \mathrm{~ms}$ post-stimulus. The onset $(50 \mathrm{~ms})$ represents the earliest artifact-free data that was reliably recorded post-stimulus. The offset $(150 \mathrm{~ms})$ represents the mean dura- 
tion of $\mathrm{GABA}_{\mathrm{B}}$ receptor mediated inhibitory neurotransmission that is recorded neurophysiologically (Fitzgerald et al., 2009) and is related to callosal inhibitory mechanisms (Daskalakis et al., 2002). An average interhemispheric transfer time of $10 \mathrm{~ms}$ was chosen to account for the time it takes for the signal to propagate from the site of the stimulation to the contralateral hemisphere (Ferbert et al., 1992). Therefore, in the right hemisphere, the area under the rectified curve was obtained between 60 and $160 \mathrm{~ms}$ post-stimulus.

Equation 1:

$\%$ ISP $=\left[\frac{\text { Area under rectified curve }(\text { Right Cortex })}{\text { Area under rectified curve }(\text { Left Cortex })}\right] \times 100$

\section{STATISTICAL ANALYSIS}

Two tailed paired $t$-tests were used to examine the difference between the cortical evoked potentials in the left and right hemispheres for both motor cortex and DLPFC. Pearson correlation coefficients were used to assess the relationship between the five personality domains of NEO-R with TMS-induced ISP in motor cortex and DLPFC. Non-parametric Spearman correlation coefficients were also calculated give the small sample size and the non-continuous NEO-FFI factors. To account for multiple comparisons, a Bonferroni corrected alpha $=0.01$ was applied. All statistical analyses were performed using SPSS 15.0 (SPSS Inc. Chicago, IL, USA).

\section{RESULTS \\ TMS-INDUCED ISP \\ Dorsolateral prefrontal cortex}

Consistent with previous findings (Voineskos et al., 2010), the activation of the right DLPFC was significantly lower compared to the cortical evoked activity in left DLPFC (right: $581.8 \pm 334.1 \mu \mathrm{V} / \mathrm{ms}$, left: $974.8 \pm 487.6 \mu \mathrm{V} / \mathrm{ms} ; t=2.9, \mathrm{df}=9, p=0.018)($ Figure $2 \mathrm{~A})$. The mean TMS-induced ISP, measured through Eq. 1, was $66.6 \pm 37.2 \%$ across subjects. The activation of the ipsi- and contralateral hemisphere is also demonstrated in the topographic illustration of the averaged cortical evoked responses measured at various latencies (Figure 3A).

\section{Motor cortex}

Consistent with previous findings (Voineskos et al., 2010), the activation of the right motor cortex was significantly lower compared to the cortical evoked activity in left motor cortex (right: $284.3 \pm 131.8 \mu \mathrm{V} / \mathrm{ms}$, left: $717.9 \pm 453.2 \mu \mathrm{V} / \mathrm{ms} ; t=3.8, \mathrm{df}=9$, $p=0.004$ ) (Figure 2B). The mean TMS-induced ISP, measured through Eq. 1, was $48.2 \pm 21.4 \%$. Similar to DLPFC, the activation of both left and right hemispheres is shown in the topographic illustration of cortical evoked potentials (Figure 3B).

\section{THE RELATIONSHIP BETWEEN NEO-FFI AND TMS-INDUCED ISP}

We found a significant relationship between TMS-induced ISP in DLPFC, and Agreeableness $(r=0.78, p=0.007)$ (Figure 1A). This relationship was specific to DLPFC as no relationship was found between agreeableness and the TMS-induced ISP in the motor cortex $(r=-0.097, p=0.79)$ (Figure 1B). Tested non-parametrically,
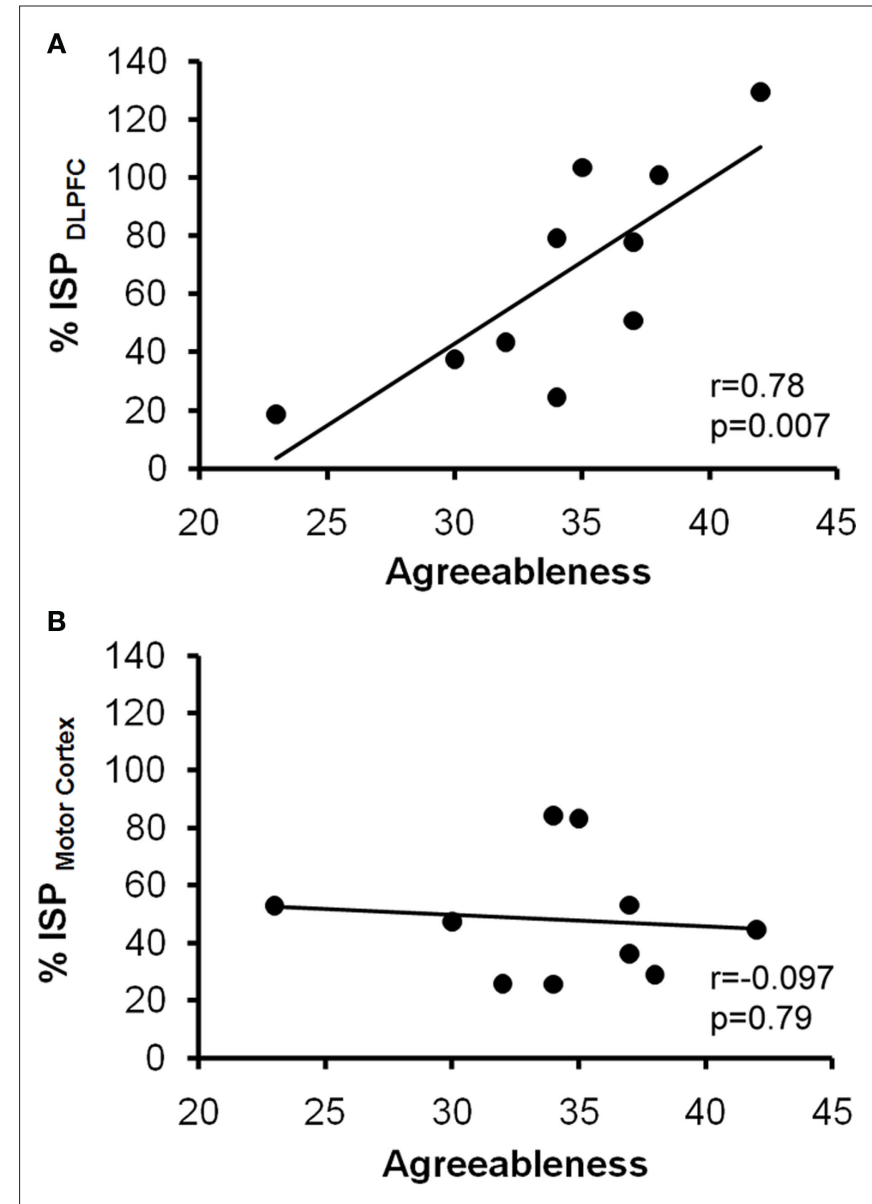

FIGURE 1 |The Relationship between interhemispheric signal propagation and Agreeableness. Data obtained from ten healthy subjects. The $x$-axes represent the score on Agreeableness, a domain of the NEO-FFI. The $y$-axes illustrate the TMS-induced interhemispheric signal propagation (ISP) from the left DLPFC to the right DLPFC (A), and from the left motor cortex to the right motor cortex (B) obtained through equation 1.

ISP measured from the DLPFC correlated significantly with Agreeableness ( $\rho=0.80, p=0.006$ ) whereas ISP measured from the motor cortex was also not significant $(\rho=-0.085, p=0.815)$. In addition, Bonferroni corrected for multiple comparisons, no relationship was found between the remaining domains of NEOFFI and TMS induced ISP in the motor cortex or DLPFC, all p's $>0.01$.

\section{DISCUSSION}

This represents the first study to relate personality traits to neurophysiological indices of interhemispheric brain communication. Specifically, we aimed to elucidate the relationship between personality and prefrontal interhemispheric connectivity and demonstrate here that of the five main personality traits it was Agreeableness that related to TMS-induced ISP over the DLPFC. Importantly, this association was not found for connectivity between the motor cortices and therefore appears specific to prefrontal interhemispheric connectivity. As noted earlier, TMSinduced ISP over the DLPFC correlates with the microstructural 

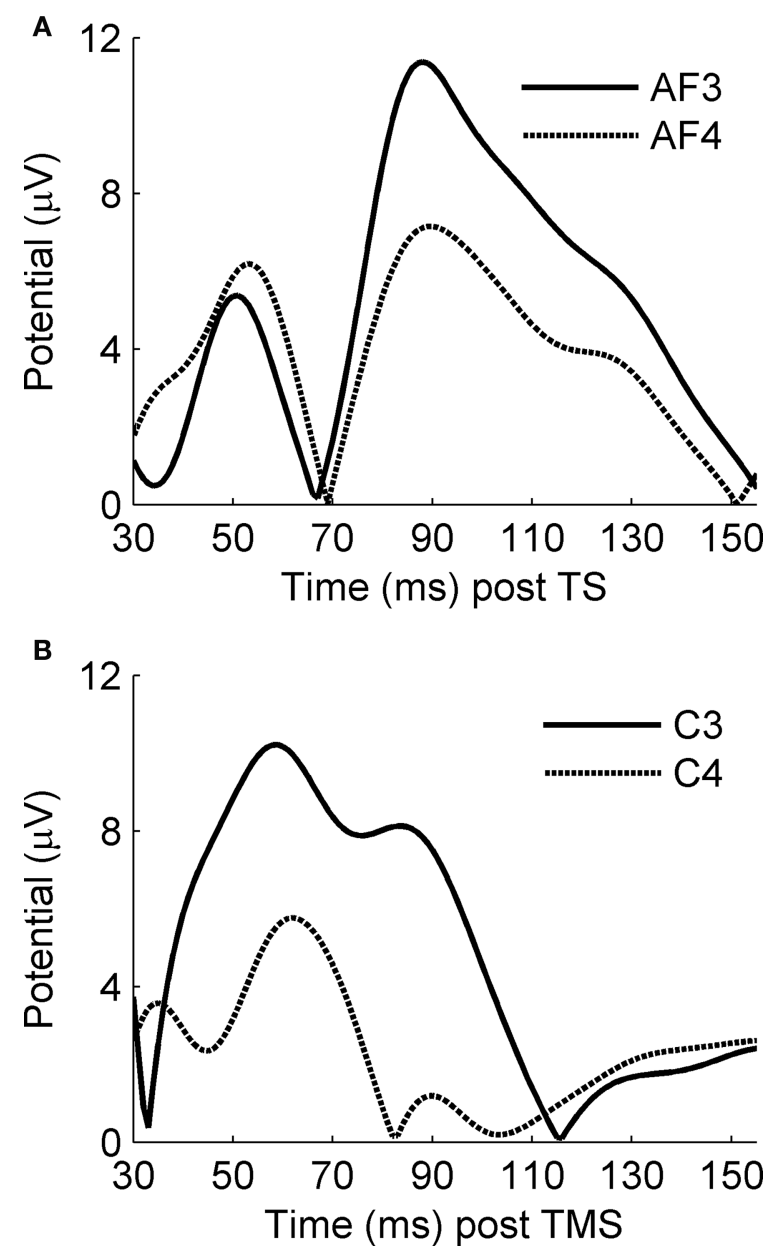

FIGURE 2 | Cortical evoked potentials in ipsi- and contralateral hemispheres following the application of TMS to the left DLPFC and left motor cortex. The waveforms represent mean rectified cortical potentials following the delivery of single pulse of TMS to the left DLPFC (A) and the left motor cortex (B). In both figures, $x$-axis represents the time after the delivery of the TMS, and the $y$-axis represents the cortical evoked potentials $(\mathrm{mV})$. These figures illustrate that application of single pulse TMS to the left hemisphere (solid waveforms) results in cortical evoked potentials in the contralateral hemisphere (dashed waveforms), that are of lower amplitude than the cortical evoked potential in the ipsilateral hemisphere.

integrity of the anterior part of the corpus callosum, the genu. These data, therefore, suggest that Agreeableness relates to the integrity of prefrontal interhemispheric connectivity that was previously shown to be mediated by the fibers in the genu of the corpus callosum. Complementary to dti, TMS-induced ISP provides information about the effective connectivity between certain brain areas. In addition, brain responses to TMS pulses measured with EEG can be divided into frequency bands providing information about the type of brain activity.

Agreeableness reflects the extent to which a person exhibits friendly, considerate and prosocial behavior aimed at cooperation and social harmony (Pereira et al., 2008; DeYoung et al., 2010). As a superordinate trait, Agreeableness covers various cognitive and emotional abilities such as empathy (Graziano et al., 2007; Nettle, 2007) and perspective-taking (Nettle and Liddle, 2008). Previous studies have shown that Agreeableness correlates with volumes of brain areas such as the superior temporal sulcus and the (posterior part of) the cingulate cortex that process information about the intentions and mental states of others (DeYoung et al., 2010). In addition, Nettle and Liddle (2008) established a positive relationship between Agreeableness and perspectivetaking. Interestingly, involved in an extensive social cognition network subserving social behavior (Frith and Frith, 1999; Decety and Jackson, 2004; Ochsner et al., 2004), the left DLPFC is associated with the processing of social information and the subsequent enabling of the prosocial behavior that is intrinsic to Agreeableness. In fact, perspective-taking, the cognitive ability to take on the perspective of another person, is dependent upon left DLPFC glutamatergic projections (Montag et al., 2008) which are also involved in interhemispheric connectivity (Daskalakis et al., 2002). Specifically, Montag et al. (2008) observed a negative correlation between glutamate levels and perspective-taking in the left DLPFC and hypothesized that the role of the DLPFC in social behavior may lie in the suppression of egoistic motivations and self-perspective (Montag et al., 2008). In line, the behavioral inhibition system (BIS) and behavioral activation system (BAS) have also been associated with the NEO-PI-R (Smits and De Boeck, 2006). In this study, Agreeableness was shown to correlate positively with BIS and negatively with a subfactor of BAS (Smits and De Boeck, 2006). Corroborating the hypothesis of Montag et al. (2008), Smits and De Boeck (2006) argue that these correlations also express the suppression of egoistic reward-driven motivations leading to more favorable behavior. The present study suggests that the left DLPFC is indeed related with prosocial behavior but adds to this understanding by demonstrating that this relationship involves communication with the contralateral homologous region. In addition, it is not unlikely that this relationship subserves the process of perspective-taking which has been shown to be positively associated with Agreeableness (Nettle and Liddle, 2008).

Psychiatric diseases in which interhemispheric connectivity is affected may be interpreted as demonstrating that interhemispheric transfer underlies certain psychiatric symptoms some of which may also be present in personality disorders. Indeed, deficits in interhemispheric connectivity have been associated with psychiatric disorders such as ADHD (Hoeppner et al., 2008) and MDD (Bajwa et al., 2008). In fact, in ADHD deficits in interhemispheric connectivity are restored by methylphenidate treatment (Hoeppner et al., 2008). Also, personality disorders have been shown to relate to deviant scores on the NEO-PI-R. For instance, in psychopathy, Agreeableness has been shown to be significantly lower (Lynam and Gudonis, 2005) and the gold standard for the diagnosis of psychopathy, the revised psychopathy checklist (PCL-R) (Hare, 2003), correlates negatively with Agreeableness (Pereira et al., 2008), demonstrating that a high degree of psychopathy is associated with little agreeableness. At present, given that our data suggest that low ISP signifies low Agreeableness, (Voineskos et al., 2010) it could be predicted that the higher degrees of psychopathy relate to a lower ISP index, although such findings need to be demonstrated directly. 


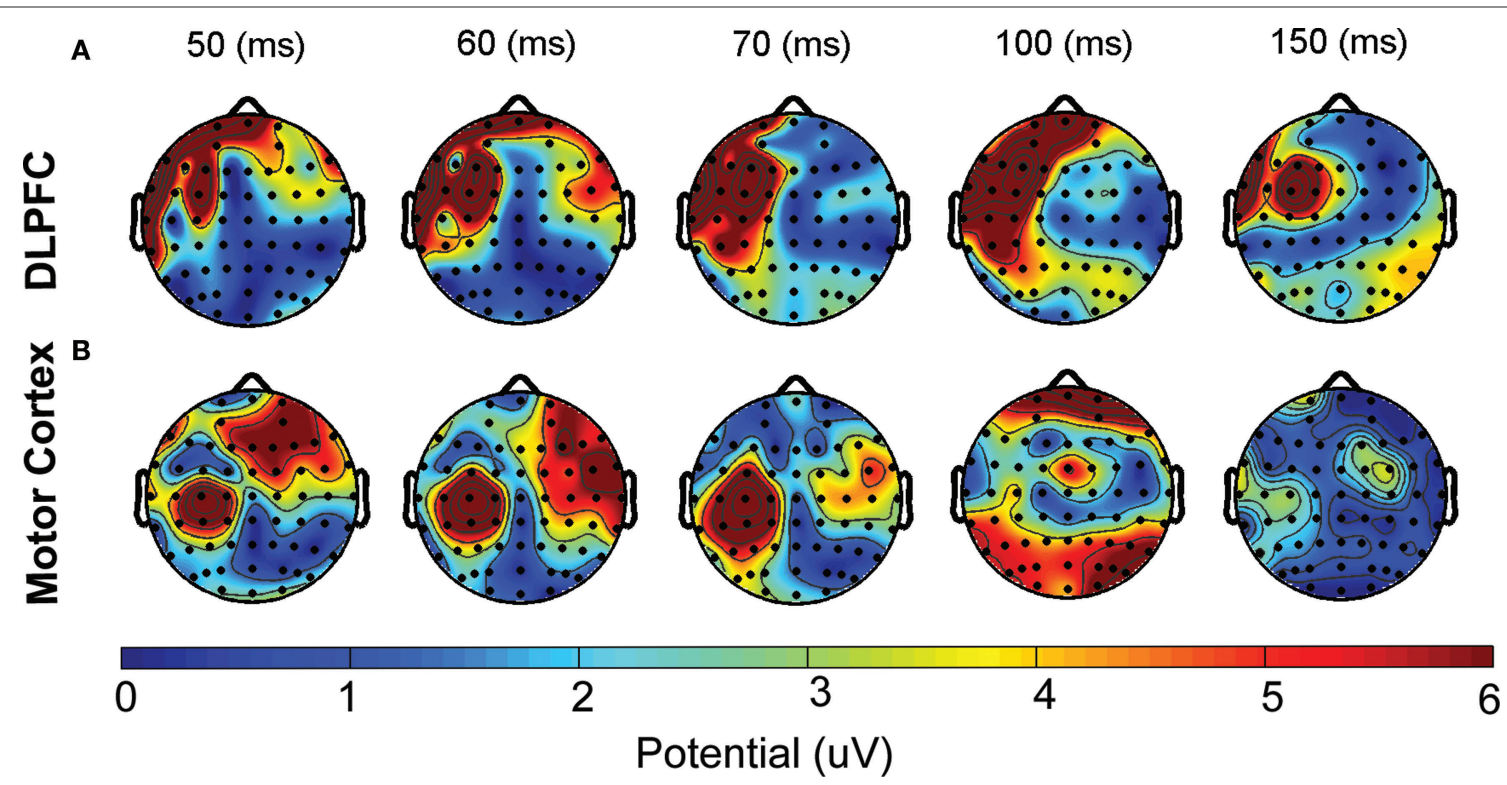

FIGURE 3 |Topographic illustration of TMS-induced signal propagation in DLPFC and motor cortex. Topographic plots illustrate the mean amplitude of cortical evoked activity in response to application of TMS single pulse to the left DLPFC (top panel) and left motor cortex (bottom panel) at 50, 60, 70, 100 and 150 ms following the TMS delivery averaged across all ten subjects. These plots suggest that, application of TMS to the left hemisphere results in activation of regions on the contralateral hemisphere but the activity is lower than that of the ipsilateral hemisphere. Topographic head plots were obtained by EEGLAB toolbox (Delorme and Makeig 2004).
The present study has some limitations. First, although the currently observed correlation was strongly significant, it should be replicated in a larger sample. In addition, the proposed hypothesis of perspective-taking and prefrontal interhemispheric connectivity may also be verified in a larger sample. Second, only left-to-right ISP was tested and as hemispheric asymmetries in relation to certain personality features have been reported (van Honk and Schutter, 2006; Hofman and Schutter, 2009), future studies may also look into right-to-left ISP. Third, we currently only indexed prefrontal interhemispheric connectivity at suprathreshold stimulus intensity levels and consequently, subthreshold stimulation should also be assayed in future testing. Despite these limitations, this study represents one of the first efforts to directly evaluate the neurophysiological relationship between connectivity in the DLPFC with personality and suggests that Agreeableness relates to the effectiveness of prefrontal communication between hemispheres. Despite these limitations, this study represents one of the first efforts to directly evaluate the neurophysiological relationship between connectivity in the DLPFC with personality and suggests that prefrontal communication between hemispheres participates in the constitution of the personality trait Agreeableness.

\section{REFERENCES}

Allport, G. (1961). Pattern and Growth in Personality. New York: Holt, Rinehart $\&$ Winston.

Bajwa, S., Bermpohl, F., Rigonatti, S. P., Pascual-Leone, A., Boggio, P. S., and Fregni, F. (2008). Impaired interhemispheric interactions in patients with major depression. J. Nerv. Ment. Dis. 196, 671-677.

Beaulieu, C. (2002). The basis of anisotropic water diffusion in the nervous system - a technical review. NMR. Biomed. 15, 435-455.

Daskalakis,Z.J.,Christensen,B.K., Fitzgerald, P. B., Roshan, L., and Chen, R. (2002). The mechanisms of interhemispheric inhibition in the human motor cortex. J. Physiol. 543(Pt 1), 317-326.

Daskalakis, Z. J., Farzan, F., Barr, M. S., Maller, J. J., Chen, R., and Fitzgerald,
P. B. (2008a). Long-interval cortical inhibition from the dorsolateral prefrontal cortex: a TMS-EEG study. Neuropsychopharmacology 33, 2860-2869.

Daskalakis, Z. J., Farzan, F., Barr, M. S, Rusjan, P. M, Favalli, G., Levinson, A. J., and Fitzgerald, P. B. (2008b). Evaluating the relationship between long interval cortical inhibition, working memory and gamma band activity in the dorsolateral prefrontal cortex. Clin. EEG Neurosci. 39, 150-155.

Decety, J., and Jackson, P. L. (2004). The functional architecture of human empathy. Behav. Cogn. Neurosci. Rev. 3, 71-100.

DeYoung, C. G., Hirsh, J. B., Shane, M. S, Papademetris, X., Rajeevan, N., and Gray, J. R. (2010). Testing predictions from personality neuroscience: brain structure and the big five. Psychol. Sci. 21, 820-828.

Doron, K.W., and Gazzaniga, M.S. (2008). Neuroimaging techniques offer new perspectives on callosal transfer and interhemispheric communication. Cortex 44, 1023-1029.

Farzan, F., Barr, M. S., Wong, W., Chen, R., Fitzgerald,P.B.,andDaskalakis,Z.J.(2009). Suppression of gamma-oscillations in the dorsolateral prefrontal cortex following long interval cortical inhibition: a TMSEEGstudy.Neuropsychopharmacology34, 1543-1551.

Ferbert, A., Priori, A., Rothwell, J. C., Day, B. L., Colebatch, J. G., and Marsden, C. D. (1992). Interhemispheric inhibition of the human motor cortex. J. Physiol. $453,525-546$.

Fitzgerald, P. B., Maller, J. J., Hoy, K., Farzan, F., and Daskalakis, Z. J. (2009).
GABA and cortical inhibition in motor and non-motor regions using combined TMS-EEG: a time analysis. Clin. Neurophysiol. 120, 1706-1710.

Frith, C. D., and Frith, U. (1999). Interacting minds - a biological basis. Science 286, 1692-1695.

Frokjaer,V.G., Mortensen, E. L., Nielsen, F. A., Haugbol, S., Pinborg, L. H., Adams, K. H., Svarer, C., Hasselbalch, S. G., Holm, S., Paulson, O. B., and Knudsen, G. M. (2008). Frontolimbic serotonin $2 \mathrm{~A}$ receptor binding in healthy subjects is associated with personality risk factors for affective disorder. Biol. Psychiatry 63, 569-576.

Graziano, W. G., Habashi, M. M., Sheese, B. E., and Tobin, R. M. (2007). Agreeableness, empathy, and helping: a person $\mathrm{x}$ situation perspective. J. Pers. Soc. Psychol. 93, 583-599. 
Hare, R. D. (2003). Manual for the Hare Psychopathy Checklist-Revised, 2nd Edn. Toronto: Multi-Health Systems.

Herwig, U., Satrapi, P., and SchönfeldtLecuona, C. (2003). Using the international 10-20 EEG system for positioning of transcranial magnetic stimulation. Brain Topogr. 16, 95-99.

Hoeppner, J., Wandschneider, R., Neumeyer, M., Gierow, W., Haessler, F., Herpertz, S. C., and Buchmann, J. (2008). Impaired transcallosally mediated motor inhibition in adults with attention-deficit/hyperactivity disorder is modulated by methylphenidate. J. Neural Transm. 115, 777-785.

Hofman, D., and Schutter, D. J. (2009). Inside the wire: aggression and functional interhemispheric connectivity in the human brain. Psychophysiology. 46, 1054-1058.

Lynam, D. R., and Gudonis, L. (2005). The development of psychopathy. Annu. Rev. Clin. Psychol. 1, 381-407.

McAdams, D. P., and Olson, B. D. (2010). Personality development: continuity and change over the life course. Annu. Rev. Psychol. 61, 517-542.

McCrae, R. R. (1991). The five-factor model and its assessment in clinical settings. J. Pers. Assess. 57, 399-314.

McCrae, R. R., and Costa, P. T. Jr., and Martin, T. A. (2005). The NEOPI-3: a more readable revised NEO Personality Inventory. J. Pers. Assess. 84, 261-270.

Montag, C., Schubert, F., Heinz, A., and Gallinat, J. (2008). Prefrontal cortex glutamate correlates with mental perspective-taking. PLoS ONE 3:e3890. doi: 10.1371/journal.pone.0003890.
Nettle, D. (2007). Empathizing and systemizing: what are they, and what do they contribute to our understanding of psychological sex differences? $\mathrm{Br}$. J. Psychol. 98(Pt 2), 237-255.

Nettle,D.,andLiddle,P.(2008).Agreeableness is related to social-cognitive but not social-perceptual, theory of mind. Eur. J. Pers. 22, 323-335.

Ochsner, K. N., Knierim, K., Ludlow, D. H., Hanelin, J., Ramachandran, T., Glover, G., and Mackey, S.C. (2004). Reflecting upon feelings: an fMRI study of neural systems supporting the attribution of emotion to self and other. J. Cogn. Neurosci. 16, 1746-1772.

Oldfield, R.C. (1971). The assessment and analysis of handedness: the Edinburgh inventory. Neuropsychologia 9, 97-113.

Pereira, N., Huband, N., and Duggan, C. (2008). Psychopathy and personality. An investigation of the relationship between theNEO-Five Factor Inventory (NEO-FFI) and the Psychopathy Checklist-Revised (PCL-R) in a hospitalized sample of male offenders with personality disorder. Crim. Behav. Ment. Health 18, 216-223.

Roberts, B. W., Walton, K. E., and Viechtbauer, W. (2006). Patterns of mean-level change in personality traits across the life course: a meta-analysis of longitudinal studies. Psychol. Bull. $132,1-25$.

Rossini, P. M., Barker, A. T., Berardelli, A., Caramia, M. D., Caruso, G., Cracco, R. Q., Dimitrijević, M. R., Hallett, M., Katayama, Y., and Lücking, C. H. (1994). Non-invasive electrical and magnetic stimulation of the brain, spinal cord and roots: basic principles and procedures for routine clinical application. Report of an IFCN committee. Electroencephalogr. Clin. Neurophysiol. 91, 79-92.

Rusjan, P. M., Barr, M. S., Farzan, F. Arenovich, T., Maller, J. J., Fitzgerald, P. B., and Daskalakis, Z. J. (in press) Optimal transcranial magnetic stimulation coil placement for targeting the dorsolateral prefrontal cortex using novel magnetic resonance imageguided neuronavigation. Hum. Brain Mapp. doi: 10.1002/hbm.20964.

Schutter, D. J., de Weijer, A. D., Meuwese, J. D., Morgan, B., and van Honk, J. (2008). Interrelations between motivational stance, cortical excitability, and the frontal electroencephalogram asymmetry of emotion: a transcranial magnetic stimulation study. Hum. Brain Mapp. 29, 574-580.

Smits, D., and De Boeck, P. (2006). From BIS/BAS to the big five. Eur. J. Pers. 20, 255-270.

Tauscher, J., Bagby, R. M., Javanmard, M., Christensen, B. K., Kasper, S., and Kapur, S. (2001). Inverse relationship between serotonin 5-HT(1A) receptor binding and anxiety: a $[(11) \mathrm{C}]$ WAY-100635 PET investigation in healthy volunteers. Am. J. Psychiatry 158, 1326-1328.

van Honk, J., and Schutter, D. J. (2006) From affective valence to motivational direction: the frontal asymmetry of emotion revised. Psychol. Sci. 17, 963-965.

Voineskos, A., Farzan, F., Barr, M. S. Lobaugh, N. J., Mulsant, B. H., Chen, R., Fitzgerald, P. B., and Daskalakis, Z. J.
2010). The role of the corpus callosum in TMS-induced interhemispheric signal propagation. Biol. Psychiatry doi: 10.1016/j.biopsych.2010.06.021.

Wassermann, E. M., Greenberg, B. D., Nguyen, M. B., and Murphy, D. L. (2001). Motor cortex excitability correlates with an anxiety-related personality trait. Biol. Psychiatry 50, 377-382.

Conflict of Interest Statement: The authors declare that the research was conducted in the absence of any commercial or financial relationships that could be construed as a potential conflict of interest.

Received: 08 August 2010; accepted: 26 September 2010; published online: 22 November 2010.

Citation: Hoppenbrouwers SS, Farzan F, Barr MS, Voineskos AN, Schutter DJLG, Fitzgerald PB and Daskalakis ZJ (2010) Personality goes a long a way: an interhemispheric connectivity study. Front. Psychiatry 1:140. doi: 10.3389/ fpsyt.2010.00140

This article was submitted to Frontiers in Neuropsychiatric Imaging and Stimulation, a specialty of Frontiers in Psychiatry. Copyright (c) 2010 Hoppenbrouwers, Farzan, Barr, Voineskos, Schutter, Fitzgerald and Daskalakis. This is an open-access article subject to an exclusive license agreement between the authors and the Frontiers Research Foundation, which permits unrestricted use, distribution, and reproduction in any medium, provided the original authors and source are credited. 